\title{
Analysis of the theoretical current-voltage characteristic of non-stationary transport in the cross-section of the desalination channel
}

\author{
I Shkorkina ${ }^{1}, N$ Chubyr $^{2}, V$ Gudza $^{1}$ and $M$ Urtenov $^{1, *}$ \\ ${ }^{1}$ Kuban State University, Krasnodar, 350040, Russia \\ ${ }^{2}$ Kuban State Technological University, Krasnodar, 350072, Russia
}

\begin{abstract}
In practice, the current-voltage characteristic (CVC) is the most important characteristic of transport in electromembrane systems, since it is using CVC that the concept of limiting current is introduced, various modes of operation of electromembrane systems are analyzed, and their efficiency is evaluated. At present, experimental CVC methods of Fourier analysis, wavelet analysis, and dynamical systems are well studied. At the same time, the study of theoretical CVC is not sufficiently developed. Previously, we derived a formula for calculating the CVC of a nonstationary 1:1 transfer of an electrolyte in the cross-section of the desalination channel, which includes an anion-exchange (AEM) and cation-exchange (CEM) membranes, and establishing the fundamental laws of changes in CVC over time. The simulation is based on the NernstPlanck-Poisson equations. In this paper, we analyze this formula and identify the fundamental laws of the CVC of non-stationary 1:1 transfer of the electrolyte in the cross-section of the desalination channel. It is shown that in the prelimiting mode, the migration current and the diffusion current give approximately the same contribution to the total current, and in the overlimiting mode, the main contribution is given by the migration current, the value of the displacement current does not depend on time and is proportional to the sweep speed. It is found that the average conduction current is many times greater than the displacement current, starting from a few seconds. The results obtained allow to construct and analyze the CVC for the cross-section of the desalination channel.
\end{abstract}

\section{Introduction}

Electromembrane methods, including electrodialysis (ED) [1], are the most profitable processes in the field of providing fresh water to millions of people, and they have already proven their environmental and economic feasibility [2]. Therefore, the development and research of mathematical models $[3,4]$ of various electrochemical phenomena in electromembrane systems (ED systems) is an actual task. To evaluate the efficiency and performance of ED systems, the current-voltage characteristic (CVC) is used. For its

\footnotetext{
* Corresponding author: urtenovmax@mail.ru
} 
calculation it is necessary to derive a formula for on the basis of some mathematical model. In this paper, we use our mathematical model of 1:1 electrolyte transfer in the cross-section of the desalination channel and a formula for calculating the CVC that is stable with respect to rounding errors. We analyze this formula and identify the fundamental laws of the CVC of non-stationary 1:1 transfer of the electrolyte in the cross-section of the desalination channel in this paper.

Thus, the scientific novelty of the work lies in the fact that, first, the section of the desalination channel is considered, and not the diffusion layer [5, 6]. Secondly, for the first time, the relations between the various components of the total current are established, for example, it is established that the average conduction current is many times greater than the displacement current, starting from a few seconds. In addition, it is shown that at prelimiting mode, the migration current and diffusion current give approximately the same contribution to the total current, and the overlimiting mode gives the main contribution of migration current, the magnitude of the displacement current is independent of time and proportional to the speed of the sweep potential.

\section{Mathematical model}

As a boundary value problem for calculating the CVC, we use a non-stationary onedimensional mathematical model of mass transfer of a binary electrolyte [6]:

$$
\begin{gathered}
\frac{\partial C_{i}}{\partial t}=-\frac{\partial j_{i}}{\partial x}, \quad i=1,2 \\
j_{i}=-\frac{F}{R T} z_{i} D_{i} C_{i} \frac{\partial \varphi}{\partial x}-D_{i} \frac{\partial C_{i}}{\partial x}, \quad i=1,2 \\
\frac{\partial^{2} \varphi}{\partial x^{2}}=-\frac{F}{\varepsilon_{a}}\left(z_{1} C_{1}+z_{2} C_{2}\right) \\
I_{c}=F\left(z_{1} j_{1}+z_{2} j_{2}\right)
\end{gathered}
$$

Here (1) are the material balance equations, (2) are the Nernst - Planck equations for the flows of sodium $i=1 \leftrightarrow \mathrm{Na}^{+}$and chlorine ions $i=2 \leftrightarrow \mathrm{Cl}^{-}$, the charge numbers of cations $z_{1}=1$ and anions $z_{2}=-1$, (3) is the Poisson equation for the electric field potential, (4) is the current flow equation, which means that the current flowing through the cross section of the desalination channel is determined by the ion flow. Constants: $\varepsilon_{a}-$ dielectric permittivity of the solution, $F$ - Faraday number, and $R$ is the universal gas constant. The desired functions: $\varphi$ - potential, $E=-\frac{\partial \varphi}{\partial x}-$ electric field strength, $C_{i}, j_{i}, D_{i}, I_{c}$ - concentration, flow, diffusion coefficient of the i-th ion, current density determined by the ion flow respectively.

The boundary conditions have the form:

For $x=0$ : 


$$
\begin{gathered}
\left.\left(\frac{F}{R T} C_{1} \frac{\partial \varphi}{\partial x}+\frac{\partial C_{1}}{\partial x}\right)\right|_{x=0}=0 \\
C_{2}(t, 0)=C_{2 m} \\
\varphi(t, 0)=\Delta_{r} \varphi=d \cdot t
\end{gathered}
$$

For $x=H$, where $H$ is the cross - section width of the desalination channel:

$$
\begin{gathered}
C_{1}(t, H)=C_{1 m} \\
\left.\left(\frac{F}{R T} C_{2} \frac{\partial \varphi}{\partial x}-\frac{\partial C_{2}}{\partial x}\right)\right|_{x=H}=0 \\
\varphi(t, H)=0
\end{gathered}
$$

where $d$ is the scanning speed over time of the potential difference. The constant $d$ has the dimension $V / s$.

Boundary conditions (5) and (9) mean ideal selectivity of ion-exchange membranes. The values of ion concentrations passing through the membranes at the boundary points are the values of the exchange capacities of ion-exchange membranes.

A potentiodynamic mode is considered, where the potential jump (7) changes over time.

Constant initial conditions are added to the boundary conditions:

$$
C_{1}(0, x)=C_{0}, C_{2}(0, x)=C_{0}, \varphi(0, x)=0
$$

Determine the average current density:

$$
I_{a v}(t)=\frac{1}{H} \int_{0}^{H} I_{c}(t, x) d x
$$

\section{Analysis of the ratio of total and partial CVC. Dependence of the CVC on the scanning speed of the potential difference}

From (1-4) follows [3]: $I_{c}=I_{m i g}+I_{\text {diff }}$, where $I_{m i g}=\frac{F^{2}}{R T_{0}}\left(z_{1}^{2} D_{1} C_{1}+z_{2}^{2} D_{2} C_{2}\right) E$ is the electromigration current, $I_{\text {diff }}=-\frac{F \cdot \partial\left(z_{1} D_{1} C_{1}+z_{2} D_{2} C_{2}\right)}{\partial x}$ - diffusion current. We determine the average electromigration and diffusion currents:

$$
\begin{gathered}
I_{m i g, a v}(t)=\frac{F^{2}}{R T_{0} H} \int_{0}^{H}\left(z_{1}^{2} D_{1} C_{1}+z_{2}^{2} D_{2} C_{2}\right) E(t, x) d x \\
I_{\text {diff,av }}(t)=-F \frac{z_{1} D_{1} C_{1}(t, H)-z_{1} D_{1} C_{1}(t, 0)+z_{2} D_{2} C_{2}(t, H)-z_{2} D_{2} C_{2}(t, 0)}{H}
\end{gathered}
$$


We denote $I_{\text {lim }}$ the limiting diffusion current ( $I_{\text {lim }}=0.30407 \mathrm{~A} / \mathrm{m}^{2}$ ) [3].

Figure 1a shows that for «large» values $d$, for small values of the potential jump «splash» occur, which then quickly fade out and the CVC has a classical form (the magnitude of the «splash» and the time to enter the quasi-stationary mode depends on the value $d$ ). The «splash» is associated with the effects of non-stationarity at large $d$ [4]. For «small» values $d$, starting from $10^{-6} \mathrm{~V} / \mathrm{s}$, the CVC is practically independent of the value $d$.

Figure $1 \mathrm{~b}$ shows that in the prelimiting mode, the migration current and the diffusion current give approximately the same contribution to the total current, and in the overlimiting mode, the main contribution is given by the migration current.

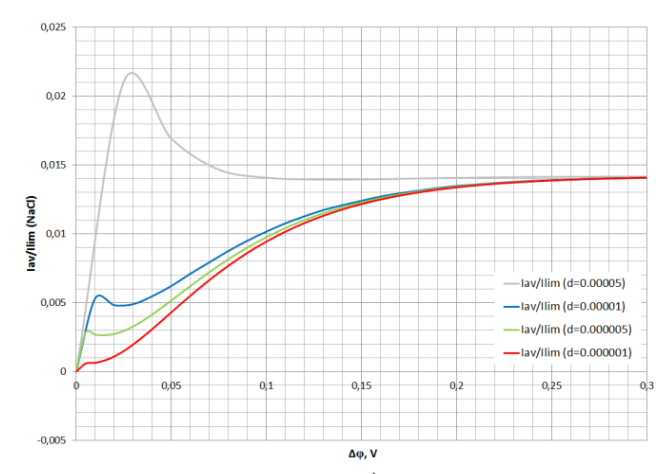

a)

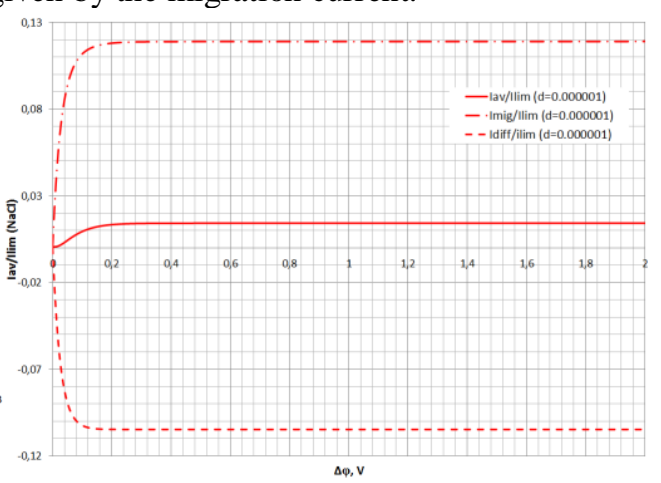

b)

Fig. 1. Graphs of $\mathrm{CVC}$ for $\mathrm{NaCl}$ solution. The ordinate shows the relative values of the current density $i_{a v}(t) / i_{\text {lim }}$ on the abscissa is the potential jump in volts: a) dependence on scanning speed in time of the potential difference, $b$ ) comparison of average current with average currents of migration and diffusion CVC graphics.

\section{Relation of the sweep speed of the potential difference and the average displacement current}

Consider the average width of the channel displacement current

$$
I_{d, a v}(t)=\frac{\varepsilon_{r}}{H} \int_{0}^{H} \frac{\partial E(t, x)}{\partial t} d x
$$

Since $\varphi_{d}(t)=-d \cdot t$, we get:

$$
I_{d, a v}(t)=\frac{\varepsilon_{r}}{H} \int_{0}^{H} \frac{\partial E(t, x)}{\partial t} d x=-\frac{\varepsilon_{r}}{H} \frac{\partial}{\partial t} \int_{0}^{H} \frac{\partial \varphi(t, H)}{\partial x} d x=-\frac{\varepsilon_{r}}{H} \frac{\partial}{\partial t}(\varphi(t, H)-\varphi(t, 0))=\frac{\varepsilon_{r}}{H} d
$$

The value $I_{d, a v}(t)=\frac{\varepsilon_{r} d}{H}$ is independent of $t$ and shows that the average displacement current is proportional to the scanning speed. 
Average conduction current $I_{a v}(t)$, for many times greater than the displacement current, starting from a few seconds. For example, $I_{d, a v}=7 \cdot 10^{-5} \mathrm{~A} / \mathrm{m}^{2}$ or $\frac{I_{d, a v}}{I_{\lim }} \approx 0.23 \cdot 10^{-3}$ at $d=0.01 \mathrm{~V} / \mathrm{s}$.

\section{Conclusion}

In this paper, a mathematical model is constructed for non-stationary 1:1 transfer of an electrolyte in the cross-section of the desalination channel in the potentiodynamic mode, and the formula (11) for calculating the CVC is stable with respect to rounding errors. The fundamental laws of the CVC of non-stationary 1:1 electrolyte transfer in the cross-section of the desalination channel are revealed.

The relations between the total current and the conduction and displacement currents are analyzed. It is shown that in the prelimiting mode, the migration current and the diffusion current give approximately the same contribution to the total current, and in the overlimiting mode, the main contribution is given by the migration current, the value of the displacement current does not depend on time and is proportional to the scanning speed. It is found that the average conduction current is many times greater than the displacement current, starting from a few seconds.

\section{Acknowledgments}

The reported study was funded by RFBR, project number 19-08-00252 A "Theoretical and experimental study of current-voltage characteristics of electromembrane systems".

\section{References}

1. Pruyn K T, Harrington J J and Smith J D 1969 Mathematical Model of the Electrodialysis Process (Cincinnati, Ohio: Department of the Interior. Federal Water Quality Admin)

2. Carolin C F, Kumar P S, Saravanan A, Joshiba G J and Naushad M 2017 J. Environ. Chem. Eng. 5 2782-2799 doi:10.1016/j.jece.2017.05.029

3. Nikonenko V, Urtenov M, Mareev S and Pourcelly G 2020 Membranes 10(2) 22

4. Mareev S A, Evdochenko E, Wessling M, Kozaderova O A, Niftaliev S I, Pismenskaya N D and Nikonenko V V 2020 Journal of Membrane Science $\mathbf{6 0 3}$ 118010

5. Chubyr N O, Gudza V A, Urtenov M A Kh and Shkorkina I V 2020 International Journal of Mechanical and Production Engineering Research and Development 10(3) 4757-4764

6. Uzdenova A, Kovalenko A, Urtenov M and Nikonenko V 2018 Membranes 8(3) 84 doi:10.3390/membranes8030084 